\title{
Existence of a bounded approximate identity in a tensor product
}

\section{David A. Robblins}

\begin{abstract}
It has been shown that the existence of a (left) approximate identity in the tensor product $A \otimes_{\alpha} B$ of Banach algebras $A$ and $B$, where $\alpha$ is an admissible algebra norm on $A \otimes B$, implies the existence of approximate identities in $A$ and $B$. The question has been raised as to whether the boundedness of the approximate identity in $A \otimes_{\alpha} B$ implies the boundedness of the approximate identities in $A$ and $B$. This paper answers the question affirmatively with $\alpha$ being the greatest cross-norm.
\end{abstract}

Loy has shown [1] that, for arbitrary Banach algebras $A$ and $B$, if $A \otimes_{\alpha} B$ has a left (right) approximate identity, then so do $A$ and $B$, where $\alpha$ is any admissible algebra norm on $A \otimes B$. He raises the question of whether existence of a bounded approximate identity in $A \otimes_{\alpha} B$ would ensure the boundedness of the approximate identities in $A$ and $B$. We are able to answer this question affirmatively when $\alpha$ is the greatest cross-norm (which we denote by $\gamma$ ), as follows:

THEOREM. Let $A$ and $B$ be Banach algebras, and let $A \otimes, B$ have a bounded (left) approximate identity. Then both $A$ and $B$ have bounded

Received 10 November 1971. The author was partially supported by a National Science Foundation Traineeship during this research. An addendum to the original paper was received 1 February 1972, and has been used by the Editor to shorten the paper, by substituting most of the addendum for most of the original paper. 
(Zeft) approximate identities.

Proof. Let $\left\{\sum_{i=1}^{\infty} a_{i}^{(j)} \otimes b_{i}^{(j)}\right\}_{j \in J}$ be a bounded left approximate identity in $A \otimes_{\gamma} B$, with $\left\|\sum_{i=1}^{\infty} a_{i}^{(j)} \otimes b_{i}^{(j)}\right\| \leq C$ for all $j \in J$.

Fix $b \in B$ such that $\|b\|=I$. Choose $G \in B^{*}$ such that $\|G\|=G(b)=1$. By properties of the norm $\gamma[2, \mathrm{p} .36]$, we have that $\sum_{i=1}^{\infty} G\left(b_{i}^{(j)} b\right) a_{i}^{(j)} \in A$. for all $j \in J$, and, moreover, that the net $\left\{\sum_{i=1}^{\infty} G\left\{b_{i}^{(j)} b\right) a_{i}^{(j)}\right\}_{j \in J}$ is bounded in $A$, for

$$
\begin{aligned}
\left\|\sum_{i=1}^{\infty} G\left(b_{i}^{(j)} b\right) a_{i}^{(j)}\right\| & \leq\|G\|\left\|_{i=1}^{\infty} a_{i}^{(j)} \otimes b_{i}^{(j)} b\right\| \\
& \leq\|b\|\left\|_{i=1} a_{i}^{(j)} \otimes b_{i}^{(j)}\right\| \leq c .
\end{aligned}
$$

We claim that $\left\{\sum_{i=1}^{\infty} G\left(b_{i}^{(j)} b\right) a_{i}^{(j)}\right\}_{j \in J}$ is a left approximate identity in $A$ : let $a \in A$. Then, again depending on the properties of $\gamma$, we have

$$
\begin{aligned}
\left\|\sum_{i=1}^{\infty} G\left(b_{i}^{(j)} b\right) a_{i}^{(j)} a-a\right\| & =\left\|\sum_{i=1}^{\infty} G\left\{b_{i}^{(j)} b\right\} a_{i}^{(j)} a-G(b) a\right\| \\
& \leq\left\|\sum_{i=1}^{\infty} a_{i}^{(j)} a \otimes b_{i}^{(j)} b-a \otimes b\right\| \\
& =\left\|\left(\sum_{i=1}^{\infty} a_{i}^{(j)} \otimes b_{i}^{(j)}\right\}(a \otimes b)-a \otimes b\right\| \stackrel{j}{\longrightarrow},
\end{aligned}
$$

so that our net is a left approximate identity in $A$.

The situation is obviously symetric with respect to $B$. 


\section{References}

[1] R.J. Loy, "Identities in tensor products of Banach algebras", Bull. Austral. Math. Soc. 2(1970), 253-260.

[2] Robert Schatten, A theory of cross-spaces (Annals of Mathematics Studies, 26. Princeton University Press, Princeton, New Jersey, 1950).

\section{Ouke University,}

Durham,

North Carolina,

USA. 